\title{
The timing of cognitive plasticity in physiological aging: a tDCS study of naming
}

\author{
Anna Fertonani ${ }^{1}$, Michela Brambilla ${ }^{1}$, Maria Cotelli ${ }^{1}$ and Carlo Miniussi ${ }^{1,2 *}$ \\ ${ }^{1}$ Cognitive Neuroscience Section, IRCCS Istituto Centro San Giovanni di Dio Fatebenefratelli, Brescia, Italy \\ ${ }^{2}$ Neuroscience Section, Department of Clinical and Experimental Sciences, University of Brescia, Brescia, Italy
}

\section{Edited by: \\ David Bartrés-Faz, University of \\ Barcelona, Spain}

\section{Reviewed by:}

Aurel Popa-Wagner, Clinic of

Psychiatry, Germany

Pablo Billeke, Universidad del

Desarrollo, Chile

${ }^{*}$ Correspondence:

Carlo Miniussi, Department of Clinical and Experimental Sciences,

University of Brescia, Viale Europa

11, 25123 Brescia, Italy

e-mail: carlo.miniussi@

cognitiveneuroscience.it
This study aimed to explore the effects of transcranial direct current stimulation (tDCS) on physiologically aging adults performing a naming task. tDCS is a method that modulates human cortical excitability. Neuroplasticity is considered to have its foundation in cortical excitability as a property that adjusts the connection strength between neurons in the brain. Language efficiency, as all functions, relies on integration of information (i.e., effectiveness of connectivity) through neurons in the brain. So the use of tDCS, to modulate cortical excitability, can help to define the state of cognitive plasticity in the aging brain. Based on Hebb's rule, an increase in synaptic efficacy does not rely only on the increase of excitability but also on the timing of activation. Therefore, a key issue in this study is the timing of tDCS application in relation to a task: When to deliver tDCS to induce modulatory effects on task execution to facilitate naming. Anodal tDCS was applied to the left dorsolateral prefrontal cortex of older and young adults before and during a naming task. In older adults, tDCS improved naming performance and decreased the verbal reaction times only if it was applied during the task execution, whereas in young subjects both stimulation conditions improved naming performance. These findings highlight that in healthy aging adults, the cerebral network dedicated to lexical retrieval processing may be facilitated only if stimulation is applied to an "active" neural network. We hypothesize that this change is due to the neuronal synaptic changes, in the aging brain, which reduce the window of when cortical excitability can facilitate synaptic efficacy and therefore plasticity.

Keywords: aging, language, transcranial direct current stimulation, facilitation, neuroplasticity, NIBS

\section{INTRODUCTION}

Language is a critical cognitive function for the communication processes in humans, and it is vital to successful social functioning. In general, the effect of physiological aging on language is characterized by a complex pattern of alterations (Cotelli et al., 2012), and evidences suggest that normal aging selectively impairs certain language abilities more than others. Although older adults know more words than young adults (Kemper and Sumner, 2001), they are more likely to experience difficulty that is manifested as slowing in producing words while speaking (Kemper, 2006; Burke and Shafto, 2008). Notable declines in these kinds of language processing undermine older adults' abilities and desire to communicate which promotes withdrawal from social interaction (Hummert et al., 2004), that might favor a more general cognitive decline (Palmer, 1990). Conversely, the beneficial role of an enriched environment in promoting the plasticity in aging has now been demonstrated. Studies on aged rodents showed that an enriched environment increases neurogenesis (Speisman et al., 2013), and significantly improved the rate and extent of stroke recovery (Buchhold et al., 2007). Studies focusing on word production by employing picture-naming tasks have demonstrated an age-related decline in object and action naming (Goodglass, 1980; Nicholas et al., 1985; LaBarge et al., 1986; Ardila and Rosselli, 1989; Feyereisen, 1997). Among others, a work by Goral et al. (2007) supports the hypothesis that the word retrieval declines, during healthy aging (Burke and Mackay, 1997; Thornton and Light, 2006; Burke and Shafto, 2008) suggesting an important role of prefrontal structures in these tasks (Cabeza, 2002).

Recent evidences have suggested that brain modifications underlying the decline of cognitive functions (associated with physiological aging) are not caused by neuronal loss; rather, they are associated with alterations in synaptic connectivity (Pakkenberg et al., 2003; Morrison and Baxter, 2012). These alterations are more pronounced in anterior than in posterior brain regions, and they reach a maximum level in the prefrontal cortex (Davis et al., 2008). One influential hypothesis focuses on the role of the dendritic spines at the dorsolateral prefrontal cortex (DLPFC) level; the hypothesis specifically references thin spines, whose numbers are apparently greatly reduced during healthy aging (Peters et al., 2008; Dumitriu et al., 2010). Therefore, the efficacy of cognitive performance and plasticity mechanisms may be altered during healthy aging.

Transcranial direct current stimulation (tDCS) is a technique that utilizes the application of a very low direct current (Priori, 2003; Nitsche et al., 2008; Paulus, 2011). During the delivery of the current (i.e., online stimulation), tDCS modulates the resting membrane potential of neurons in a direction that depends 
on the polarity (anodal vs. cathodal) of the electrode placed on the chosen area. Since the first studies (Nitsche and Paulus, 2001; Nitsche et al., 2003b), the presence of offline effects (effects that persist beyond the stimulation period) has been highlighted in addition to the online effects (during stimulation). Online and offline effects seem mediated by different mechanisms. It has been showed that online anodal tDCS-induced effects are related to membrane depolarization because they are effected by ion-channel blocking substances (Stagg and Nitsche, 2011), while offline tDCS-induced effects involve the additional participation of glutamatergic N-methyl-D-aspartic (NMDA) receptors and therefore a long-term potentiation-like (LTP-like) mechanism (Liebetanz et al., 2002; Stagg and Nitsche, 2011). Therefore, changes in duration of the induced effect should depend on change on one of these mechanisms.

Several studies have recently applied anodal tDCS with the aim of highlighting the presence of behavioral facilitator effects on several cognitive functions including language (see Monti et al., 2012 for a recent review). For example, Fertonani et al. (2010) observed a behavioral facilitation in a naming task after the application of anodal tDCS on the left DLPFC. This facilitatory function performed by tDCS may be important in cognitive neurorehabilitation approaches because the technique can improve reduced cognitive abilities in several neurological pathologies. Nevertheless, one caveat is that most of these studies were conducted on young healthy adults, and the generalization of the effects to other subjects (particularly patients who are not in the same age range of younger adult subjects) is not guaranteed (see Holland and Crinion, 2012). From this perspective, the physiological aging brain represents an interesting model to study and to predict result of tDCS applications in the clinical field; generally, patients that enter a neurorehabilitation approach are not young and therefore their brain is similar to the brain of a healthy older adult. Understanding what happens when tDCS is applied to an aged brain would validate the application of transcranial electric stimulation methods in a population that could maximally benefit from the enhancement of residual abilities.

For these reasons, researchers have recently become interested in the application of tDCS to older adults (Holland et al., 2011; Ross et al., 2011). Nevertheless, some issues must be clarified before using tDCS in rehabilitative protocols. An aspect that has not been adequately investigated in previous studies is the optimal time to apply tDCS when testing cognitive function. Some recent studies have suggested that when to deliver tDCS in relation to a task of interest may represent a key variable in determining the effects of tDCS (Stagg et al., 2011; Pirulli et al., 2013, 2014). An interesting work by Wirth et al. (2011) examined the behavioral and electrophysiological effects of online and offline anodal tDCS during a naming task. The behavioral effect was present only with online application. After anodal offline tDCS there was not behavioral effect but was present a reduction in delta activity, interpreted as a sign of neural disinhibition.

If neuroplasticity is reliant on the degree of neuronal excitability in a given moment as an element that adjusts the strength of neuronal connections in the brain, the timing of the tDCS application and when this "treatment" induces neuronal effects on task execution are important issues. The present work aims to explore the effects of tDCS in healthy aging adults performing picture-naming tasks by investigating the ideal moment for applying anodal stimulation (i.e., during or before the execution of the task). In general, tDCS may "prime" the system by (i) increasing excitability by modifying the synaptic "weights" of the system via LTP-like mechanisms that imply the involvement of NMDA receptors or (ii) increasing the excitability of the stimulated area by modulating axon, dendrite, and soma resting potentials and eventually increasing the quantity of neurotransmitters released from the presynaptic neuron without inducing longterm effects. Both of these hypotheses predict facilitation under specific conditions. The first hypothesis suggest that tDCS can induce facilitation mainly during offline applications via LTP-like mechanisms, but also during online stimulation via membrane depolarization (Liebetanz et al., 2002). The second hypothesis suggests facilitation only during online tDCS. We hypothesize that in the healthy aging brain, the mechanisms of plasticity subtending offline stimulation effects may be altered due to neuronal dysfunction (Pakkenberg et al., 2003; Morrison and Baxter, 2012). Consequently, the offline facilitation effects of tDCS should be less pronounced than the online effects. We also tested a group of young healthy subjects to investigate the possibility of different behavioral effects, induced by a different neural efficiency, in the two groups of age.

\section{METHODS \\ SUBJECTS}

Twenty young subjects ( 10 males; mean age 21.2 years, standard deviation 0.9 , range $20-23$, mean education 13.0 years) participated in the Experiment on young. Twenty healthy-aging adults (10 males, mean age 66.5 years, standard deviation 5.5, range 6183, mean education 10.5 years) participated in the Experiment on elderly.

The subjects were right-handed native Italian speakers with normal or corrected-to-normal vision. We did not include subjects who had a history of seizures, implanted metal objects, heart problems or any other neurological or psychiatric disease. Healthy aged individuals who scored below 27 out of 30 on the Mini Mental State Examination (MMSE) were also excluded. In addition, the elderly participants were subjected to a complete, accurate neuropsychological evaluation; a pathological score in at least one of the tests was a further exclusion criterion. The neuropsychological test battery included measures to assess non-verbal reasoning (Raven Colored Progressive Matrices), language comprehension (Token test), verbal fluency (phonemic and semantic), the object-/action-naming and comprehension subtests of the Battery for the Analysis of the Aphasic Deficit (BADA) (Miceli et al., 1994), memory (Story Recall, Rey-Osterrieth Complex Figure recall, Digit Span, Spatial Span), visuo-spatial abilities (Rey-Osterrieth Complex Figure, copy), attention and executive functions (Trail-Making test A and B). The results of the cognitive assessment are presented in Table 1.

The study was approved by the Ethics Committee of IRCCS Centro San Giovanni di Dio Fatebenefratelli, Brescia, Italy. Informed consent was obtained from all participants prior to the beginning of the experiment. 
Table 1 | Neuropsychological data (mean) of the elderly participants.

\begin{tabular}{|c|c|c|}
\hline & Raw score & Cut-off \\
\hline \multicolumn{3}{|l|}{ SCREENING FOR DEMENTIA } \\
\hline MMSE $^{a}$ & $29.0 / 30$ & 24 \\
\hline \multicolumn{3}{|l|}{ NON-VERBAL REASONING } \\
\hline Raven colored progressive matrices & $29.5 / 36$ & 17.5 \\
\hline \multicolumn{3}{|l|}{ MEMORY } \\
\hline Story recall & $14.1 / 28$ & 7.5 \\
\hline Rey-Osterrieth complex figure, recall & $14.2 / 36$ & 9.46 \\
\hline Digit span & 5.9 & 3.75 \\
\hline Spatial span & 5.1 & 3.55 \\
\hline \multicolumn{3}{|l|}{ PRAXIA } \\
\hline Rey-Osterrieth complex figure, copy & $32.1 / 36$ & 28.87 \\
\hline \multicolumn{3}{|l|}{ EXECUTIVE FUNCTIONS } \\
\hline Trail-making test A (seconds) & 36.4 & 93 \\
\hline Trail-making test B (seconds) & 108.2 & 282 \\
\hline \multicolumn{3}{|l|}{ LANGUAGE } \\
\hline Token test & $34.0 / 36$ & 26.5 \\
\hline Fluency, phonemic & 39.1 & 16 \\
\hline Fluency, semantic & 47.3 & 24 \\
\hline Oral object comprehension (BADA ${ }^{b}$ ) & $39.9 / 40$ & \\
\hline Oral action comprehension (BADA ${ }^{b}$ ) & $19.8 / 20$ & \\
\hline Oral object naming (BADA $\left.{ }^{\mathrm{b}}\right)$ & $29.5 / 30$ & \\
\hline Oral action naming (BADA ${ }^{b}$ ) & $27.6 / 28$ & \\
\hline
\end{tabular}

${ }^{a}$ Mini mental state examination

${ }^{b}$ Battery for the analysis of the aphasic deficit.

\section{PICTURE-NAMING TASK}

The stimuli for the picture-naming task were presented on a personal computer screen using the Presentation v. 12.0 software program (http://www.neurobs.com). All of the stimuli were black-and-white, two-dimensional line drawings from the corpus of the CRL-IPNP (http://crl.ucsd.edu/ aszekely/ipnp), a broad set of 795 action and object pictures. These items have been tested in healthy and patient populations in seven different international sites and languages. The items are coded for a number of variables known to influence naming difficulty, including initial word frequency, age of acquisition and picture imageability scores. These variables have been previously tested to assess their influence on the participants' naming performance (Bates et al., 2000).

The picture-naming task consisted of a practice block and three experimental blocks. The practice block included three object and three action images. Each experimental block included 14 object and 14 action images selected from a larger data set, which were tested in previously published behavioral experiments (Cotelli et al., 2010). We have constructed two different version of the task, to be used accordingly to the age of the experimental subjects (young, or elderly). It has been demonstrated that elderly subjects are slower than young subjects in naming pictures (Burke and Shafto, 2004). In the elderly task both the duration of stimulus presentation and the inter-stimulus interval were longer. This could potentially have affected our results slowing down the reaction times in the aging group (see e.g., Nakata et al., 2005), nevertheless, the presence of a sham condition (see below) assures us the possibility to verify if the effect is due to the stimulation or to this variable. The frequencies and lengths of the target words and the visual complexity and imageability of the pictures were matched between the blocks.

The subjects were required to accurately and rapidly name the stimuli appearing on the computer screen. The duration of presentation of each image and of the interval between the trials was longer for elderly subjects to obtain a comparable task difficulty in the two age groups. The trial structure is illustrated in Figure 1.

\section{TRANSCRANIAL DIRECT CURRENT STIMULATION}

The stimulation was delivered by a battery-driven, constantcurrent stimulator (BrainStim, EMS, Bologna, Italy) through a pair of saline-soaked sponge electrodes $(7 \times 5 \mathrm{~cm})$. A constant current of $2 \mathrm{~mA}$ was applied with a ramping period of $10 \mathrm{~s}$ both at the beginning and at the end of the stimulation. In the online condition, the current was turned on at the beginning of the task and off at the end of the task, resulting in four or 5-min-long stimulation (respectively for young and elderly participants, see Figure 1). In the offline condition, the stimulation duration was set to $10 \mathrm{~min}$ to assure the permanence of the stimulation effects during the execution of the subsequent naming task. The current density $\left(0.057 \mathrm{~mA} / \mathrm{cm}^{2}\right)$ was maintained below the safety limits (Poreisz et al., 2007; Nitsche et al., 2008). The electrodes were firmly attached by elastic bands, and an electroconductive gel was applied under the electrodes to reduce contact impedance before the montage. The active electrode was placed on the left DLPFC, $8 \mathrm{~cm}$ frontally and $6 \mathrm{~cm}$ laterally with respect to the scalp vertex (Fertonani et al., 2010), which had been identified as CZ in 10-20 nomenclature for EEG electrode positioning. The reference electrode was fixed on the right shoulder. We preferred an extracephalic reference to avoid interference effects from brain areas beneath the reference electrode.

The study was a single-blind experiment; the individual subjects were unaware of the type of stimulation they received, but the experimenter knew the type of stimulation. We applied the three following different types of stimulation to the left DLPFC: anodal online, anodal offline and sham. The duration of the stimulation in Experiment on young and in Experiment on elderly was respectively: 4 or $5 \mathrm{~min}$ for the anodal online condition (according to the different duration of the task on the two experiments), $10 \mathrm{~min}$ for the anodal offline condition, 6 or $7 \mathrm{~min}$ for the sham condition (beginning always $2 \mathrm{~min}$ before the beginning of the task). The different durations were due to the duration of task execution, faster in the Experiment on young (see Figure 1). In the sham stimulation (i.e., placebo), the current was turned off $10 \mathrm{~s}$ after the beginning of the stimulation (plus the duration of the fade-in and fade-out periods $=10 \mathrm{~s}$ ) and was turned on for the last $10 \mathrm{~s}$ of the stimulation period. Therefore, the subjects felt the itching sensations below the electrodes at the beginning and end of the stimulation, making this condition indistinguishable from the experimental stimulation. Indeed, to detect any differences in the perception of sensations, we asked the subjects to complete a questionnaire developed by our research group (Fertonani et al., 2010) regarding the sensations that they experienced during the different stimulation types (real vs. sham) (See Results). 


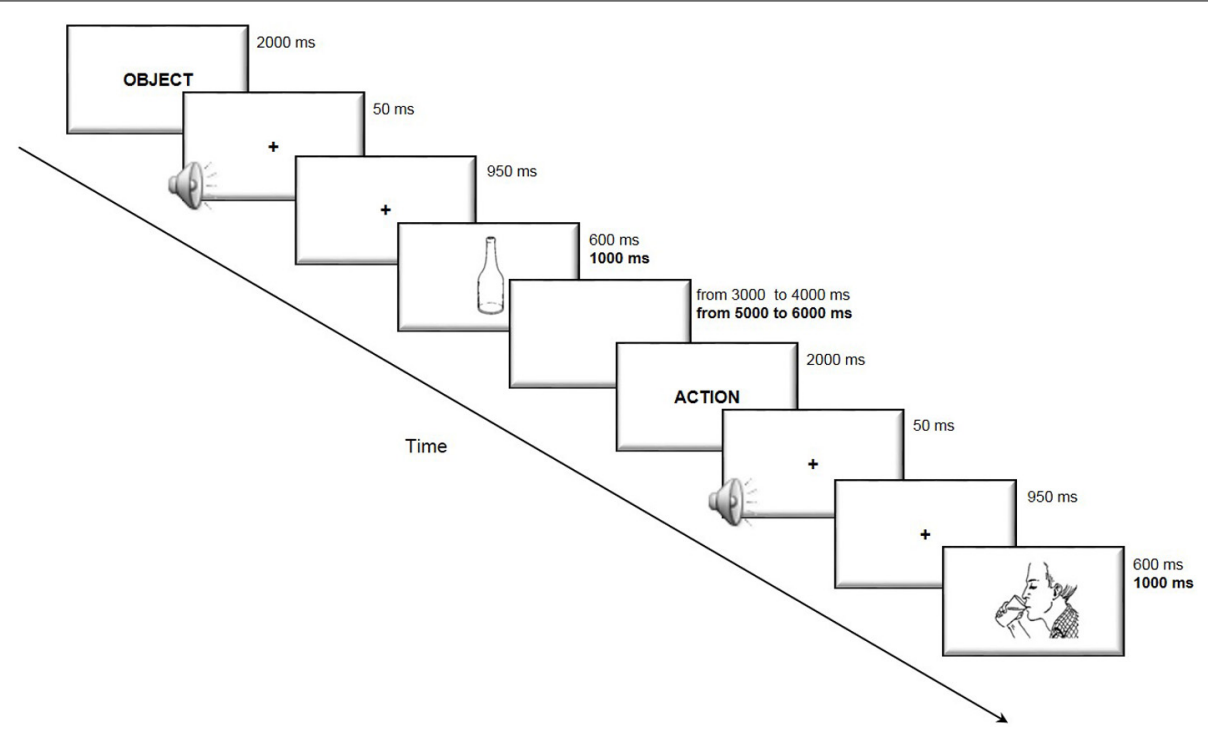

FIGURE 1 | Trial structure of the picture-naming task. The subjects were presented with an indication of the category "action" or "object" immediately before the picture was presented to disambiguate lexical selection. The participants were then required to accurately and rapidly name the stimuli appearing on the computer screen. In bold are reported the timing used in the elderly participants task version, when different from that of the young participants.

\section{PROCEDURE}

The subjects were seated in a quiet room in front of a computer screen. In the anodal online condition, the subjects performed the picture-naming task during the stimulation. In the anodal offline condition, the subjects performed the task immediately following the stimulation, and in the sham condition, the placebo stimulation began approximately 2 min before the start of the task. The active stimulations (i.e., anodal online and anodal offline) were executed on two different and consecutive days to minimize the likelihood of interference effects. The sham stimulation was always performed first on the first or second day (see Figure 2).

\section{DATA ANALYSIS}

The latency of verbal responses (vocal reaction time, vRT) and naming accuracy were determined for each subject in each condition. The subjects' answers were recorded with a microphone placed in front of the participant. The vocal responses were digitized with the GoldWave v. 5.15 software program (GoldWave, Newfoundland, Canada) with a sampling rate of $11,025 \mathrm{~Hz}$. The latency of the vRT was measured by analysing the GoldWave registration files, which marked the start of the wave corresponding to the vocal response. As standard procedure we eliminated all incorrectly performed trials from the analysis, including no responses, semantic errors, visual errors and responses preceded by verbal searching, (overall 3.2\%, that is within the normal range). In addition, we removed all vRT data above or below two standard deviations with respect to the mean for each subject in each condition.

For the vRT data, the Kolmogorov-Smirnov test confirmed the normality of the distribution; therefore, the data were subsequently analyzed using a repeated measure ANOVA. The Mauchly test was applied when appropriate to confirm the data sphericity.
Multiple comparisons were carried on using the Fisher LSD procedure.

Because the naming accuracy data were not normally distributed, we applied the appropriate non-parametric tests. A pvalue of 0.05 was considered significant for all statistical analyses except for the sensations (see below).

The data of the tDCS-induced sensations were analyzed using the Friedman test (non-parametric repeated measures comparison). The post-hoc comparisons were conducted considering a $\alpha$-value of 0.0167 ( 0.05 divided by 3 , i.e., the number of comparisons performed for each sensation).

\section{RESULTS}

We inferred that all of the subjects tolerated the stimulation by interpreting the spontaneous reports and the questionnaires completed by each subject at the end of the experiment. As evinced from verbal reports, none of the subjects could distinguish the sham from the real stimulation. The questionnaire results are reported in Table 2 . In young subjects, pitching, itchiness and burning were the most commonly reported sensations (90, 83, and $57 \%$ of the subjects, respectively), with light to moderate intensity. The Friedman test highlighted a statistically significant difference between the stimulations for the perception of itchiness $\left[\chi_{(2)}^{2}=11.472, p=0.003\right]$, pinching $\left[\chi_{(2)}^{2}=10.750, p=0.004\right]$, iron taste $\left[\chi_{(2)}^{2}=6.645, p=\right.$ $0.04]$ and effect on performance $\left[\chi_{(2)}^{2}=20.167, p<0.001\right]$. Nevertheless, post-hoc comparison showed a significant difference only between anodal online and sham condition in the itchiness, pinching and effect on performance perception (i.e., sensations greater in the anodal online condition), and between online and offline condition in the effect on performance perception. 


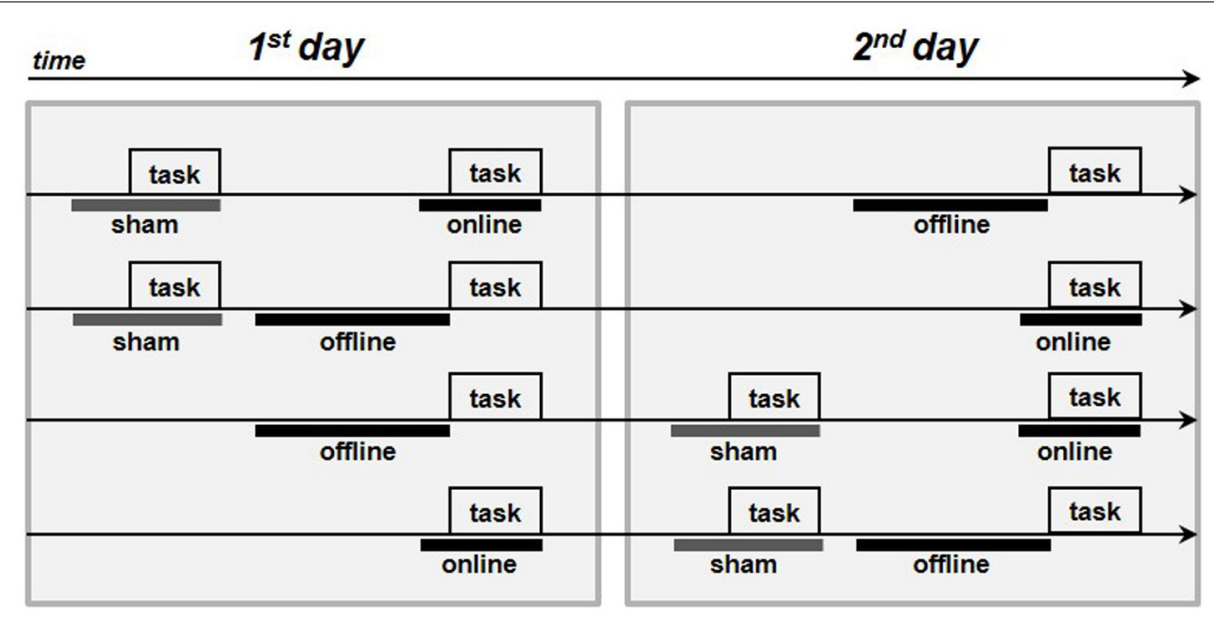

FIGURE 2 | Procedure of the experiments. The four arrows represents the four procedures adopted in each experiment. The gray boxes represent the execution of the picture-naming task, the thick gray lines represent the sham stimulation whereas the thick black lines the real tDCS stimulations (online or offline).

Table 2 | Mean intensity of the sensations reported by the subjects after transcranial direct current stimulation (tDCS) and the percentage of subjects who reported a certain sensation.

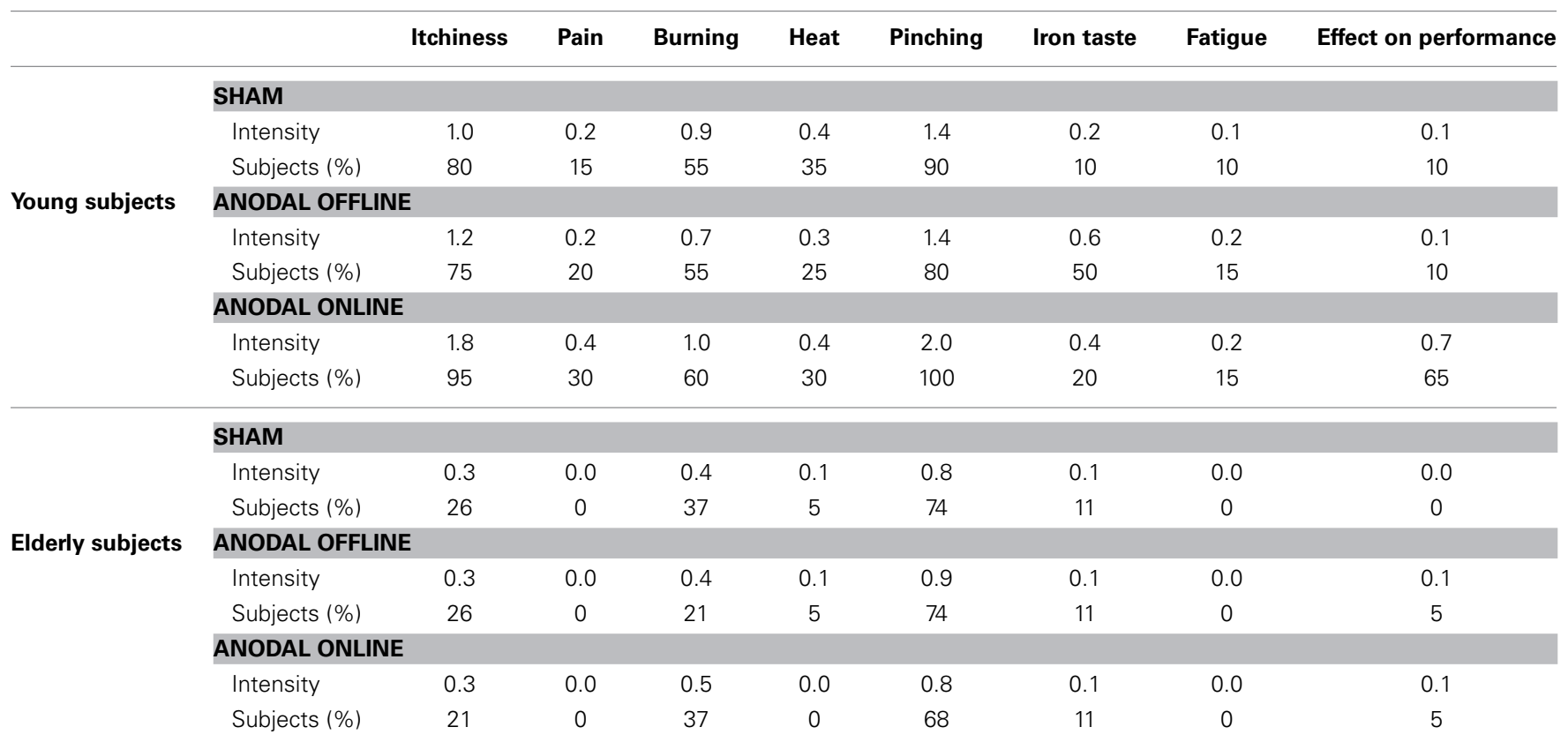

The sensation intensity is presented on a 5-point scale as follows: $0=$ None, $1=$ Mild, $2=$ Moderate, $3=$ Considerable, $4=$ Strong. The column "Effect on performance" indicates the subjective feeling of the participant relative to how much the tDCS-induced sensations affected his performance.

In elderly subjects, light-intensity pitching and burning were the most commonly reported sensations (72 and 32\% of the subjects, respectively). The Friedman test did not reveal any significant difference in the subjects' perceptions of sensation between the experimental (online or offline) or sham stimulation conditions.

\section{ACCURACY}

The accuracy level of young participants was high (96\% mean accuracy in all of the conditions, see Table 3 for more details). The accuracy did not differ among the stimulation conditions (sham, anodal online, anodal offline)[Friedman test $\left(\chi_{(2)}^{2}=1.333, p=\right.$ $0.51)]$. The Wilcoxon test on the type of stimulus (object, action) was significant $(Z=3.114, p=0.002)$. Young subjects were more accurate in the object-naming task (mean $=0.15$ errors/block) than in action-naming task ( mean $=0.83$ errors/block).

Also in the elderly group, the accuracy level was high $(97 \%$ mean accuracy in all of the conditions, see Table 3 ). The accuracy did not differ among the stimulation conditions (sham, anodal online, anodal offline $)$ [Friedman test $\left(\chi 2_{(2)}=0.634, p=\right.$ 
Table 3 | Verbal reaction time (vRT) and accuracy for young and elderly subjects in the three experimental conditions.

\section{Sham Anodal online Anodal offline}

\begin{tabular}{|c|c|c|c|c|}
\hline \multirow{6}{*}{ Young subjects } & \multicolumn{4}{|c|}{ vRT (MS) } \\
\hline & Actions & $757 \pm 72$ & $720 \pm 69$ & $710 \pm 72$ \\
\hline & Objects & $585 \pm 57$ & $578 \pm 55$ & $576 \pm 56$ \\
\hline & \multicolumn{4}{|c|}{ ACCURACY (\%) } \\
\hline & actions & $93.6 \pm 6.9$ & $93.6 \pm 6.5$ & $95.0 \pm 6.6$ \\
\hline & objects & $98.6 \pm 3.7$ & $99.3 \pm 2.2$ & $98.9 \pm 3.5$ \\
\hline & vRT (MS & & & \\
\hline & actions & $912 \pm 95$ & $871 \pm 99$ & $921 \pm 144$ \\
\hline & objects & $718 \pm 83$ & $691 \pm 65$ & $705 \pm 84$ \\
\hline \multirow[t]{3}{*}{ Elderly subjects } & \multicolumn{4}{|c|}{ ACCURACY (\%) } \\
\hline & actions & $94.6 \pm 6.9$ & $95.7 \pm 5.6$ & $94.6 \pm 8.5$ \\
\hline & objects & $98.6 \pm 2.9$ & $98.9 \pm 1.6$ & $99.6 \pm 2.6$ \\
\hline
\end{tabular}

$v R T$ are expressed as $m s \pm S D$, accuracy as percentage of accuracy $\pm S D$.

0.73)]. The Wilcoxon test on the type of stimulus (object, action) demonstrated that elderly subjects were more accurate in the object-naming task (mean $=0.15$ errors/block) than in actionnaming task (mean $=0.72$ errors/block) $(Z=2.840, p<0.01)$.

The accuracy level was not different in young and elderly group [Mann-Withney test $(Z=-1.163, p=0.245)]$. Given the very low number of errors, we did not perform further analyses on these data.

\section{RESPONSE TIMES}

We performed a repeated measures ANOVA on vRT with stimulation condition (sham, anodal online, anodal offline) and type of stimulus (object, action) as within subjects factors and age (young, elderly) as a between subjects factor. The ANOVA highlighted the main effect of age $\left[F_{(1,38)}=47.001 ; p<0.001\right.$; $\left.\eta_{P}^{2}=0.553\right]$, type of stimulus $\left[F_{(1,38)}=413.339 ; p<0.001\right.$; $\left.\eta_{P}^{2}=0.916\right]$ and stimulation condition $\left[F_{(2,76)}=6.634 ; p=\right.$ $\left.0.001 ; \eta_{P}^{2}=0.149\right]$. The interactions between stimulation condition and age $\left[F_{(2,76)}=3.305 ; p=0.042 ; \eta_{P}^{2}=0.080\right]$, and type of stimulus and age $\left[F_{(1,38)}=7.145 ; p=0.008 ; \eta_{P}^{2}=0.167\right]$ were also statistically significant.

The main effect of age demonstrates that young subjects were faster than elderly subjects (young: $654 \pm 99$ ms; elderly: $803 \pm$ $139 \mathrm{~ms}$ ). The main effect of the type of stimulus shows that subjects were faster at object naming than at action naming (objects: $578 \pm 56 \mathrm{~ms}$; actions: $713 \pm 74 \mathrm{~ms})$.

The effect of stimulation condition was better explained by the interaction stimulation condition $\times$ age. Multiple post-hoc comparisons revealed that in young there was a statistically significant difference between the sham (mean vRT $\pm S D=$ $671 \pm 109 \mathrm{~ms})$ and the anodal online $(649 \pm 94 \mathrm{~ms})$ and anodal offline $(643 \pm 93 \mathrm{~ms})$ conditions (respectively $p=0.05$ and $\mathrm{p}=$ 0.01 ; see Figure $3 \mathrm{~A}$ ), whereas in the elderly there was a statistically significant difference between the anodal online condition (781 $\pm 123 \mathrm{~ms}$ ) and the other two stimulation conditions (sham: $815 \pm 132 \mathrm{~ms}$; anodal offline: $813 \pm 159 \mathrm{~ms}$, both $p<0.01$; see Figure 3B). See Table 3 for the vRT-values in each condition.

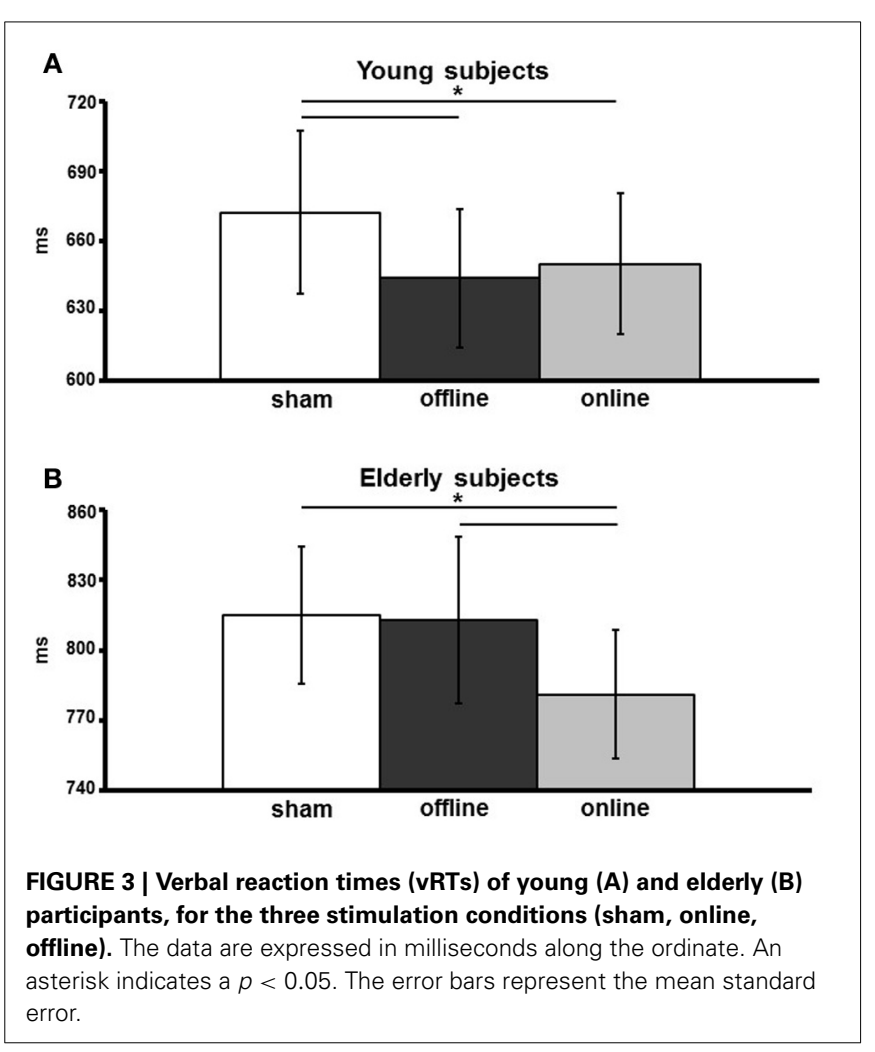

\section{DISCUSSION}

In this study, we show that anodal stimulation of the left DLPFC has a facilitation effect on picture naming in both young and healthy aging adults. In particular, whereas in young participants decreased vocal reaction times were present if the stimulation was applied both before and during the execution of the task, in the healthy aging group the effect was observed only if the stimulation was applied during the task execution. This result highlights the differential effects of tDCS in young and aging subjects. Moreover, it highlights the importance of stimulation timing in the aging group. Few studies to date have considered this variable only within the motor cortex (Nitsche et al., 2003a; Kuo et al., 2008; Stagg and Nitsche, 2011). Nitsche et al. (2003a) demonstrated that anodal tDCS applied during the execution of an implicit learning task led to an improvement in the learning rate of that task. However, if the same task was performed after 10 min of stimulation, no enhancement in the learning rate was observed (Kuo et al., 2008). Similar results have been reported by Stagg et al. (2011) in an explicit sequence-learning task. Online stimulation allows the subjects to learn more rapidly, and offline stimulation has the opposite effect, slowing learning. These results in the motor cortex are consistent with the hypothesis that anodal tDCS interacts with subsequent motor learning, in a metaplastic manner (Ziemann and Siebner, 2008), inducing decreased motor performance when tDCS is applied before the task. This mechanism works by reducing the amount of plasticity after an excitability-increasing stimulation (Bienenstock et al., 1982). Nevertheless, results in the motor cortex not always are comparable to tDCS effects induced in the cognitive domain (Jacobson et al., 2012). 
Regarding the cognitive domain, previous studies have confirmed the presence of facilitatory effects when a cognitive task is performed after anodal stimulation (offline, e.g., Ohn et al., 2008; Sparing et al., 2008; Boggio et al., 2009; Fertonani et al., 2010), and data have highlighted analogous facilitations when the task is performed during the stimulation (Fregni et al., 2005; Chi et al., 2010). Nevertheless, because these studies did not investigate both conditions, we cannot determine whether the timing of the stimulations simply differed in the degree of induced facilitation. Moreover, all of these studies were performed on young healthy adults.

Our study compared two different application timings of anodal stimulation (i.e., online vs. offline) in the same subjects who performed the same tasks, which permits us to obtain a result that allow to directly establish if the two protocols are equivalent or not. In healthy older adults, online stimulation is better than offline in inducing facilitation when the subjects are performing a picture-naming task. However, this online vs. offline difference in inducing facilitation is inconsistent with the results in the young participants. In the present experiment and in a previous published experiment from our group (Fertonani et al., 2010) we observed a facilitation in an offline condition, confirming the validity of the effect in young subjects. Such facilitation during online and offline stimulation, in young participants is in line with previous works and therefore we need to focus on differences in the elderly.

The absence of offline facilitation in the present group of older adults may be attributed to the short stimulation period (i.e., $10 \mathrm{~min}$ ). However, the short period should not be an issue because a stimulation of the same duration causes facilitation in young adults performing the same task, as we have demonstrated before (Fertonani et al., 2010) and confirmed in the present work. It is noteworthy that the neural networks of young and old adults are different, and the different responsiveness of healthy-aging neural networks may account for the absence of offline facilitation. The online facilitation is likely due to the action mechanisms of anodal stimulation, which acts on the neuronal membrane potential, making the stimulated neuronal population more excitable. These mechanisms are not efficacious enough, in healthy older adults, to induce short-term plasticity after stimulation. Studies have demonstrated that the aging brain is characterized by several structural, physiological and functional changes (Caserta et al., 2009). Studies on both humans and animals have proposed that age-related cognitive decline is more likely to be associated with alterations in synaptic connectivity, intracellular signaling and metabolism than with neuronal loss (Morrison and Baxter, 2012). In rhesus monkeys, researchers have demonstrated that the agerelated volume decrease observed in the DLPFC is attributed to a substantial synapse loss, which involves only certain synapse types (Peters et al., 2008; Dumitriu et al., 2010). Indeed, only the thin spines are involved; these spines are associated with a high degree of plasticity compared with mushroom spines. As previously reported the offline effects of a single application of tDCS have been shown to be mediated by NMDA receptor activity, the results of an LTP-like neuronal plasticity mechanisms (Liebetanz et al., 2002; Nitsche et al., 2003a). Our data suggest that, at least with the parameters adopted in this study, the stimulation of the left DLPFC in healthy aging adults cannot induce this type of plasticity; the stimulation likely intrinsically modifies the electrical properties of the neurons. This reduced plasticity induction is presumably due to the neuronal synaptic changes that involve the aging brain. $\mathrm{Ca}^{2+}$ is known to be closely involved in synaptic plasticity, particularly in triggering LTP (Fitzjohn and Collingridge, 2002). Researchers have proposed that aging is associated with an increased dysregulation of $\mathrm{Ca}^{2+}$ homeostasis (Kirischuk and Verkhratsky, 1996). We speculate that the facilitation effect of offline tDCS may be reduced by a suboptimal intracellular $\mathrm{Ca}^{2+}$ level. This level may not favor the modulation of ion channel conductance and consequent homeostasis mechanisms. tDCS is a continuous stimulation; continuous stimulation can induce neurophysiological homeostasis mechanisms, which serve to maintain neural activity within a normal functional range (Siebner et al., 2004; Siebner, 2010). Present result cannot be accounted by a slowing of the system in older adults, since the facilitatory effect was present during task execution. In this context, it is possible that in older adults, the offline stimulation protocol cannot adjust the threshold of the system due to reduced transmembrane $\mathrm{Ca}^{2+}$ conduction. Therefore, altering the intracellular $\mathrm{Ca}^{2+}$ concentrations, which is important for processes of LTP, reduces tDCS-induced after-effects. Indeed $\mathrm{Ca}^{2+}$ alteration can also modify compensatory mechanisms of metaplasticity. Given that the synaptic homeostasis is changed so will be the degree of the response to activity induce by the task and by tDCS.

In conclusion, we found that anodal stimulation applied to the left DLPFC during the execution of a picture-naming task modulates the behavioral performance of healthy aging adults. This result confirms that the left DLPFC is part of a cerebral network dedicated to lexical retrieval/selection processing in naming (Cappa et al., 2002; Cotelli et al., 2006, 2008, 2010; Fertonani et al., 2010). Crucially, the offline tDCS application, which is efficacious in young adults, did not induce facilitation effects in older adults.

It is fundamentally important to evaluate tDCS effectiveness in inducing facilitator effects in a linguistic elaboration process to facilitate the implementation of a better informed tDCS application in the neurorehabilitation field. The current study confirmed the importance of the timing choice when applying tDCS with respect to the age of experimental subjects. tDCSinduced effects are sensitive to the state of the network that is active at that moment. It has been showed that increasing cortical excitability by means of non-invasive brain stimulation may induce a reconfiguration of functional brain networks to address specific cognitive demands (Peña-Gómez et al., 2012). Thus, the polarization of neurons in combination with ongoing synaptic input can be contextualized in a framework of synaptic co-activation (Miniussi et al., 2013). Therefore the effects of tDCS should be considered in relation to the state of the cortical network carrying out a task, network that in the aging brain might present a reduced connectivity (Sala-Llonch et al., 2014). Only in this context a-tDCS-induced modulation, might primes cortical synaptic efficacy and connectivity that potentiates the system within the language network, leading to more effective processing (Vidal-Piñeiro et al., 2014). 
Based on present observations and theories (Hebb, 1949), we conclude that the capacity of an aged neural circuits to increase efficiency will be maximized when the task is executed during tDCS. Moreover, based on the same logic, learning, related to task execution, during tDCS should be more stable than learning that occurs at a different time.

\section{REFERENCES}

Ardila, A., and Rosselli, M. (1989). Neuropsychological characteristics of normal aging. Dev. Neuropsychol. 5, 307-320. doi: 10.1080/87565648909540441

Bates, E., Andonova, E., D'Amico, S., Jacobsen, T., Kohnert, K., Lu, C. C., et al. (2000). Introducing the CRL International Picture-Naming Project (CRL-IPNP). Vol. 12, La Jolla, CA: Center for Research in Language Newsletter.

Bienenstock, E. L., Cooper, L. N., and Munro, P. W. (1982). Theory for the development of neuron selectivity: orientation specificity and binocular interaction in visual cortex. J. Neurosci. 2, 32-48.

Boggio, P. S., Khoury, L. P., Martins, D. C. S., Martins, O. E. M. S., de Macedo, E. C., and Fregni, F. (2009). Temporal cortex direct current stimulation enhances performance on a visual recognition memory task in Alzheimer disease. J. Neurol. Neurosurg. Psychiatry 80, 444-447. doi: 10.1136/jnnp.2007.141853

Buchhold, B., Mogoanta, L., Suofu, Y., Hamm, A., Walker, L., Kessler, C., et al. (2007). Environmental enrichment improves functional and neuropathological indices following stroke in young and aged rats. Restor. Neurol. Neurosci. 25, 467-484.

Burke, D. M., and Mackay, D. G. (1997). Memory, language, and ageing. Philos. Trans. R. Soc. Lond. B. Biol. Sci. 352, 1845-1856. doi: 10.1098/rstb.1997.0170

Burke, D. M., and Shafto, M. A. (2004). Aging and language production. Curr. Dir. Psychol. Sci. 13, 21-24. doi: 10.1111/j.0963-7214.2004.01301006.x

Burke, D. M., and Shafto, M. A. (2008). "Language and aging," in The Handbook of Aging and Cognition, eds F. Craik and T. Salthouse (New York, NY: Psychology Press), 373-443.

Cabeza, R. (2002). Hemispheric asymmetry reduction in older adults: the HAROLD model. Psychol. Aging 17, 85-100. doi: 10.1037/0882-7974.17.1.85

Cappa, S. F., Sandrini, M., Rossini, P. M., Sosta, K., and Miniussi, C. (2002). The role of the left frontal lobe in action naming - rTMS evidence. Neurology 59, 720-723. doi: 10.1212/WNL.59.5.720

Caserta, M. T., Bannon, Y., Fernandez, F., Giunta, B., Schoenberg, M. R., and Tan, J. (2009). Normal brain aging clinical, immunological, neuropsychological, and neuroimaging features. Int. Rev. Neurobiol. 84, 1-19. doi: 10.1016/S00747742(09)00401-2

Chi, R. P., Fregni, F., and Snyder, A. W. (2010). Visual memory improved by non-invasive brain stimulation. Brain Res. 1353, 168-175. doi: 10.1016/j.brainres.2010.07.062

Cotelli, M., Manenti, R., Brambilla, M., Zanetti, O., and Miniussi, C. (2012). Naming ability changes in physiological and pathological aging. Front. Neurosci. 6:120. doi: 10.3389/fnins.2012.00120

Cotelli, M., Manenti, R., Cappa, S. F., Geroldi, C., Zanetti, O., Rossini, P. M., et al. (2006). Effect of transcranial magnetic stimulation on action naming in patients with Alzheimer disease. Arch. Neurol. 63, 1602-1604. doi: 10.1001/archneur.63.11.1602

Cotelli, M., Manenti, R., Cappa, S. F., Zanetti, O., and Miniussi, C. (2008). Transcranial magnetic stimulation improves naming in Alzheimer disease patients at different stages of cognitive decline. Eur. J. Neurol. 15, 1286-1292. doi: $10.1111 /$ j.1468-1331.2008.02202.x

Cotelli, M., Manenti, R., Rosini, S., Calabria, M., Brambilla, M., Bisiacchi, P. S., et al. (2010). Action and object naming in physiological aging: An rTMS study. Front. Aging Neurosci. 2:151. doi: 10.3389/fnagi.2010.00151

Davis, S. W., Dennis, N. A., Daselaar, S. M., Fleck, M. S., and Cabeza, R. (2008). Que PASA? The posterior-anterior shift in aging. Cereb. Cortex 18, 1201-1209. doi: 10.1093/cercor/bhm155

Dumitriu, D., Hao, J., Hara, Y., Kaufmann, J., Janssen, W. G. M., Lou, W., et al. (2010). Selective changes in thin spine density and morphology in monkey prefrontal cortex correlate with aging-related cognitive impairment. J. Neurosci. 30, 7507-7515. doi: 10.1523/JNEUROSCI.6410-09.2010

Fertonani, A., Rosini, S., Cotelli, M., Rossini, P. M., and Miniussi, C. (2010). Naming facilitation induced by transcranial direct current stimulation. Behav. Brain Res. 208, 311-318. doi: 10.1016/j.bbr.2009.10.030
Feyereisen, P. (1997). A meta-analytic procedure shows an age-related decline in picture naming: comments on Goulet, Ska, and Kahn (1994). J. Speech Lang. Hear. Res. 40, 1328-1333.

Fitzjohn, S. M., and Collingridge, G. L. (2002). Calcium stores and synaptic plasticity. Cell Calcium 32, 405-411. doi: 10.1016/\$0143416002001999

Fregni, F., Boggio, P. S., Nitsche, M., Bermpohl, F., Antal, A., Feredoes, E., et al. (2005). Anodal transcranial direct current stimulation of prefrontal cortex enhances working memory. Exp. Brain Res. 166, 23-30. doi: 10.1007/s00221005-2334-6

Goodglass, H. (1980). "Naming disorders in aphasia and aging," in Language and Comunication in the Ederly, eds L. K. Obler and M. L. Albert (Toronto, ON: Lexington Books), 37-45.

Goral, M., Spiro, A. I., Albert, M., Obler, L., and Connor, L. (2007). Change in lexical-retrieval skills in adulthood. Ment. Lex. 2, 215-240. doi: 10.1075/ml.2.2.05gor

Hebb, D. O. (1949). The Organization of Behavior: a Neuropsychological Theory. New York, NY: Wiley.

Holland, R., and Crinion, J. (2012). Can tDCS enhance treatment of aphasia after stroke?. Aphasiology 26, 1169-1191. doi: 10.1080/02687038.2011.616925

Holland, R., Leff, A. P., Josephs, O., Galea, J. M., Desikan, M., Price, C. J., et al. (2011). Speech facilitation by left inferior frontal cortex stimulation. Curr. Biol. 21, 1403-1407. doi: 10.1016/j.cub.2011.07.021

Hummert, M., Garstka, T., Ryan, E., and Bonnesen, J. (2004). "The role of age stereotypes in interpersonal communication," in Handbook of Communication and Aging Research, eds J. Nussbaum and J. Coupland (Mahwah, NJ: Routlege), 91-115.

Jacobson, L., Koslowsky, M., and Lavidor, M. (2012). tDCS polarity effects in motor and cognitive domains: a meta-analytical review. Exp. brain Res. 216, 1-10. doi: 10.1007/s00221-011-2891-9

Kemper, S. (2006). "Language in adulthood," in Lifespan Cognition: Mechanisms of Change, eds E. Bialystok and F. I. M. Craik (New York, NY: Oxford University Press), 223-238. doi: 10.1093/acprof:oso/9780195169539. 003.0015

Kemper, S., and Sumner, A. (2001). The structure of verbal abilities in young and older adults. Psychol. Aging 16, 312-322. doi: 10.1037/0882-7974.16.2.312

Kirischuk, S., and Verkhratsky, A. (1996). Calcium homeostasis in aged neurones. Life Sci. 59, 451-459. doi: 10.1016/0024-3205(96)00324-4

Kuo, M.-F. F., Unger, M., Liebetanz, D., Lang, N., Tergau, F., Paulus, W., et al. (2008). Limited impact of homeostatic plasticity on motor learning in humans. Neuropsychologia 46, 2122-2128. doi: 10.1016/j.neuropsychologia.2008. 02.023

LaBarge, E., Edwards, D., and Knesevich, J. W. (1986). Performance of normal elderly on the Boston Naming Test. Brain Lang. 27, 380-384. doi: 10.1016/0093934X(86)90026-X

Liebetanz, D., Nitsche, M. A., Tergau, F., and Paulus, W., (2002). Pharmacological approach to the mechanisms of transcranial DC-stimulation-induced aftereffects of human motor cortex excitability. Brain 125, 2238-2247. doi: 10.1093/brain/awf238

Miceli, G., Laudanna, A., Burani, C., and Papasso, R. (1994). Batteria per l'Analisi dei Deficit Afasici. B.A.D.A. (Battery for Analysis of Aphasic Deficits). Milano: CEPSAG, Università Cattolica del Sacro Cuore.

Miniussi, C., Harris, J. A., and Ruzzoli, M. (2013). Modelling non-invasive brain stimulation in cognitive neuroscience. Neurosci. Biobehav. Rev. 37, 1702-1712. doi: 10.1016/j.neubiorev.2013.06.014

Monti, A., Ferrucci, R., Fumagalli, M., Mameli, F., Cogiamanian, F., Ardolino, G., et al. (2012). Transcranial direct current stimulation (tDCS) and language. J. Neurol. Neurosurg. Psychiatry 84, 832-842. doi: 10.1136/jnnp-2012-302825

Morrison, J. H., and Baxter, M. G. (2012). The ageing cortical synapse: hallmarks and implications for cognitive decline. Nat. Rev. Neurosci. 13, 240-250. doi: $10.1038 /$ nrn 3200

Nakata, H., Inui, K., Wasaka, T., Tamura, Y., Kida, T., and Kakigi, R. (2005). Effects of ISI and stimulus probability on event-related go/nogo potentials after somatosensory stimulation. Exp. Brain Res. 162, 293-299. doi: 10.1007/s00221004-2195-4

Nicholas, M., Obler, L., Albert, M. L., and Goodglass, H. (1985). Lexical retrieval in healthy aging. Cortex 21, 595-606. doi: 10.1016/S0010-9452(58)80007-6

Nitsche, M. A., Cohen, L. G., Wassermann, E. M., Priori, A., Lang, N., Antal, A., et al. (2008). Transcranial direct current stimulation: state of the art 2008. Brain Stimul. 1, 206-223. doi: 10.1016/j.brs.2008.06.004 
Nitsche, M. A., Fricke, K., Henschke, U., Schlitterlau, A., Liebetanz, D., Lang, N., et al. (2003a). Pharmacological modulation of cortical excitability shifts induced by transcranial direct current stimulation in humans. J. Physiol. 553, 293-301. doi: 10.1113/jphysiol.2003.049916

Nitsche, M. A., Nitsche, M. S., Klein, C. C., Tergau, F., Rothwell, J. C., and Paulus, W. (2003b). Level of action of cathodal DC polarisation induced inhibition of the human motor cortex. Clin. Neurophysiol. 114, 600-604. doi: 10.1016/S13882457(02)00412-1

Nitsche, M. A., and Paulus, W. (2001). Sustained excitability elevations induced by transcranial DC motor cortex stimulation in humans. Neurology 57, 1899-1901. doi: 10.1212/WNL.57.10.1899

Ohn, S. H., Park, C.-I., Yoo, W.-K., Ko, M.-H., Choi, K. P., Kim, G.-M., et al. (2008). Time-dependent effect of transcranial direct current stimulation on the enhancement of working memory. Neuroreport 19, 43-47. doi: 10.1097/WNR.0b013e3282f2adfd

Pakkenberg, B., Pelvig, D., Marner, L., Bundgaard, M. J., Gundersen, H. J. G., Nyengaard, J. R., et al. (2003). Aging and the human neocortex. Exp. Gerontol. 38, 95-99. doi: 10.1016/S0531-5565(02)00151-1

Palmer, R. M. (1990). "Failure to thrive" in the elderly: diagnosis and management. Geriatrics 45, 47-50, 53-5.

Paulus, W. (2011). Transcranial electrical stimulation (tES - tDCS; tRNS, tACS) methods. Neuropsychol. Rehabil. 21, 602-617. doi: 10.1080/09602011.2011.557292

Peña-Gómez, C., Sala-Lonch, R., Junqué, C., Clemente, I C., Vidal, D., Bargalló, N., et al. (2012). Modulation of large-scale brain networks by transcranial direct current stimulation evidenced by resting-state functional MRI. Brain Stimul. 5, 252-263. doi: 10.1016/j.brs.2011.08.006

Peters, A., Sethares, C., and Luebke, J. I. (2008). Synapses are lost during aging in the primate prefrontal cortex. Neuroscience 152, 970-981. doi: 10.1016/j.neuroscience.2007.07.014

Pirulli, C., Fertonani, A., and Miniussi, C. (2013). The role of timing in the induction of neuromodulation in perceptual learning by transcranial electric stimulation. Brain Stimul. 6, 683-689. doi: 10.1016/j.brs.2012. 12.005

Pirulli, C., Fertonani, A., and Miniussi, C. (2014). Is neural hyperpolarization by cathodal stimulation always detrimental at the behavioral level? Front. Behav. Neurosci. 8:226. doi: 10.3389/fnbeh.2014.00226

Poreisz, C., Boros, K., Antal, A., and Paulus, W. (2007). Safety aspects of transcranial direct current stimulation concerning healthy subjects and patients. Brain Res. Bull. 72, 208-214. doi: 10.1016/j.brainresbull.2007. 01.004

Priori, A. (2003). Brain polarization in humans: a reappraisal of an old tool for prolonged non-invasive modulation of brain excitability. Clin. Neurophysiol. 114, 589-595. doi: 10.1016/S1388-2457(02)00437-6

Ross, L. A., McCoy, D., Coslett, H. B., Olson, I. R., and Wolk, D. A., (2011). Improved proper name recall in aging after electrical stimulation of the anterior temporal lobes. Front. Aging Neurosci. 3:16. doi: 10.3389/fnagi.2011.00016

Sala-Llonch, R., Junqué, C., Arenaza-Urquijo, E. M., Vidal-Piñeiro, D., VallsPedret, C., Palacios, E. M., et al. (2014). Changes in whole-brain functional networks and memory performance in aging. Neurobiol Aging. doi: 10.1016/j.neurobiolaging.2014.04.007. [Epub ahead of print].
Siebner, H. R. (2010). A primer on priming the human motor cortex. Clin. Neurophysiol. 121, 461-463. doi: 10.1016/j.clinph.2009.12.009

Siebner, H. R., Lang, N., Rizzo, V., Nitsche, M. A., Paulus, W., Lemon, R. N., et al. (2004). Preconditioning of low-frequency repetitive transcranial magnetic stimulation with transcranial direct current stimulation: evidence for homeostatic plasticity in the human motor cortex. J. Neurosci. 24, 3379-3385. doi: 10.1523/JNEUROSCI.5316-03.2004

Sparing, R., Dafotakis, M., Meister, I. G., Thirugnanasambandam, N., and Fink, G. R. (2008). Enhancing language performance with non-invasive brain stimulation-a transcranial direct current stimulation study in healthy humans. Neuropsychologia 46, 261-268. doi: 10.1016/j.neuropsychologia.2007.07.009

Speisman, R. B., Kumar, A., Rani, A., Pastoriza, J. M., Severance, J. E., Foster, T. C., et al. (2013). Environmental enrichment restores neurogenesis and rapid acquisition in aged rats. Neurobiol. Aging 34, 263-274. doi: 10.1016/j.neurobiolaging.2012.05.023

Stagg, C. J., Jayaram, G., Pastor, D., Kincses, Z. T., Matthews, P. M., and JohansenBerg, H. (2011). Polarity and timing-dependent effects of transcranial direct current stimulation in explicit motor learning. Neuropsychologia 49, 800-804. doi: 10.1016/j.neuropsychologia.2011.02.009

Stagg, C., and Nitsche, M. (2011). Physiological basis of transcranial direct current stimulation. Neuroscience 17, 37-53. doi: 10.1177/1073858410386614

Thornton, R., and Light, L. L. (2006). "Language comprehension and production in normal aging," in Handbook of the Psychology of Aging, eds J. E. Birren and K. Warner Schaie (Burlington, MA: Elsevier), 262-287.

Vidal-Piñeiro, D., Martin-Trias, P., Arenaza-Urquijo, E. M., Sala-Llonch, R., Clemente, I. C., Mena-Sánchez, I., et al. (2014). Task-dependent activity and connectivity predict episodic memory network-based responses to brain stimulation in healthy aging. Brain Stimul. 7, 287-296. doi: 10.1016/j.brs.2013.12.016

Wirth, M., Rahman, R. A., Kuenecke, J., Koenig, T., Horn, H., Sommer, W., et al. (2011). Effects of transcranial direct current stimulation (tDCS) on behaviour and electrophysiology of language production. Neuropsychologia 49, 3989-3998. doi: 10.1016/j.neuropsychologia.2011.10.015

Ziemann, U., and Siebner, H. R. (2008). Modifying motor learning through gating and homeostatic metaplasticity. Brain Stimul. 1, 60-66. doi: 10.1016/j.brs.2007.08.003

Conflict of Interest Statement: The authors declare that the research was conducted in the absence of any commercial or financial relationships that could be construed as a potential conflict of interest.

Received: 27 March 2014; accepted: 05 June 2014; published online: 24 June 2014. Citation: Fertonani A, Brambilla M, Cotelli $M$ and Miniussi $C$ (2014) The timing of cognitive plasticity in physiological aging: a tDCS study of naming. Front. Aging Neurosci. 6:131. doi: 10.3389/fnagi.2014.00131

This article was submitted to the journal Frontiers in Aging Neuroscience.

Copyright (C) 2014 Fertonani, Brambilla, Cotelli and Miniussi. This is an openaccess article distributed under the terms of the Creative Commons Attribution License (CC BY). The use, distribution or reproduction in other forums is permitted, provided the original author(s) or licensor are credited and that the original publication in this journal is cited, in accordance with accepted academic practice. No use, distribution or reproduction is permitted which does not comply with these terms. 